\title{
Regadenoson stress during low-level exercise: The EXERRT trial-does it move the needle?
}

\author{
John J. Mahmarian, MD, MASNC \\ a Houston Methodist DeBakey Heart \& Vascular Center, Houston, TX
}

Received Mar 24, 2017; accepted Mar 24, 2017

doi: $10.1007 / \mathrm{s} 12350-017-0873-4$

\section{See related article, pp. 788-802}

\section{INTRODUCTION}

The introduction of pharmacologic stressor agents over three decades ago expanded stress myocardial perfusion imaging (MPI) to the large and ever growing number of patients who were unable to achieve adequate exercise stress due to non-cardiac reasons. This is true for both single-photon emission computed tomography (SPECT) but particularly for positron emission tomography where exercise stress cannot currently be performed due to the short half-life of the radiotracers.

Subsequent advancements were the development of semi-selective adenosine A2A receptor agonists which had a more favorable side effect profile as compared to non-selective agents (e.g., Adenosine and Dipyridamole) and therefore could be safely used in stable patients with asthma and chronic obstructive pulmonary disease. ${ }^{1}$ Regadenoson (REG) is currently the only A2A agonist approved by the Federal Drug Administration for pharmacologic stress testing and it allows rapid administration of stressor and radiotracer within 1 minute with elimination of infusion pumps and weight-based dosing. All of these advances have led to greater flexibility and versatility of imaging in nuclear cardiology laboratories.

Although pharmacologic agents are ideal in patients who cannot achieve maximal exercise stress, exercise is still the preferred stressor due to the additional information provided such as exercise duration, metabolic equivalents achieved, blood pressure response, the presence and extent of electrocardiographic ischemia,

\footnotetext{
Reprint requests: John J. Mahmarian, MD, MASNC, Houston Methodist DeBakey Heart \& Vascular Center, 6550 Fannin Street, Suite 677, Houston, Texas, 77030-2717; jmahmarian@houstonmethodist.org J Nucl Cardiol 2017;24:803-8.

$1071-3581 / \$ 34.00$

Copyright (C) 2017 American Society of Nuclear Cardiology.
}

and symptoms elicited during the test. In this regard, exercise stress is generally attempted first except in patients with clear physical limitations to exercise. Up until now, if a patient did not achieve exercise goals, the treadmill test was terminated and the patient underwent a pharmacologic stress test either later that same day or was rescheduled for testing at a later date. This inevitable disruption of the daily imaging schedule leads to laboratory inefficiency and delays in patient evaluations. In this regard, it would be advantageous to immediately administer a pharmacologic stressor agent in patients who perform an inadequate, non-diagnostic exercise test rather than wait a variable time period.

\section{THE EXERRT TRIAL}

In this issue of the Journal, Thomas et al report the results of the EXERRT trial. EXERRT is the first large randomized multicenter study to address the feasibility, safety, and accuracy of administering REG early (i.e., 3 minutes) into the recovery phase of a submaximal exercise treadmill test (REG-EX) as compared to 1 hour after termination of exercise (REG-R). Patients randomized to REG-EX continued to walk on the treadmill at a speed of 1.7 down to $1.2 \mathrm{mph}$ at a $0 \%$ incline. The study enrolled 1147 stable patients with known or suspected coronary artery disease from 44 centers in the US and South America who were referred for a clinically indicated exercise or pharmacologic stress SPECT MPI and who were unable to achieve $\geq 85 \%$ of their predicted target heart rate and/or $\geq 5$ METS during exercise treadmill testing. Patients were excluded if they had unstable angina, myocardial infarction or coronary revascularization within 30 days, second or third degree AV block, left bundle branch block, or prior placement of a pacemaker or defibrillator. Eighty-six percent of patients screened could not achieve exercise goals and were randomized in a 1:1 fashion to either REG-EX or REG-R followed by a second REG study within 114 days (Fig. 1). Most patients (96\%) underwent an 
initial single-day rest/stress imaging protocol. Visual analysis of SPECT images was performed using a 17 segment model by three expert readers blinded to the imaging sequence or randomization. Akin to the ADVANCE trials which compared the non-inferiority of adenosine-REG to those of serial adenosine-adenosine results for detecting ischemia, ${ }^{2,3}$ the EXERRT trial compared serial REG-EX and REG-R to serial REG-R SPECT MPI results. The primary endpoint was noninferiority of REG-EX vs serial REG-R for inducing reversible perfusion defects (0-1 or $\geq 2$ segments). Secondary endpoints were to assess image quality, hemodynamic changes, and the safety of rapidly performing REG stress during low-level exercise.

In the primary analysis, majority agreement rates were $92 \%$ (95\% CI 89-94) for the REG-EX and $95 \%$ (95\% CI 93-97\%) for the serial REG-R groups with a difference of $-3 \%$ (95\% CI -6 to $0 \%$ )-achieving the non-inferiority lower limit of $-7.5 \%$. Secondary analysis also showed similar serial reader agreement between the two randomized groups for summed stress scores as an assessment of initial perfusion defect size and severity. Of particular interest was that irrespective of randomized group, $8.5 \%$ of patients changed from a normal to an abnormal study from the initial to the subsequent SPECT study and $5.0 \%$ of patients developed new evidence of ischemia. Regarding image quality, sub-diaphragmatic count activity was less $(p=0.019)$ and target-to-background ratios were significantly higher with REG-EX vs REG-R ( $p<0.001)$ but image quality was rated as excellent/good in $>92 \%$ of patients in both groups.

There were no major hemodynamic differences in heart rate or systolic blood pressure between the randomized groups following REG administration. There were also no significant differences in side effects experienced by patients in the two randomized groups (53\% REG-EX vs 59\% REG-R). No patients developed second or third degree AV block. More patients demonstrated ST segment depression/elevation with REG-EX (2.8\%) vs REG-R (0.6\%). Lastly, 3 of 575 patients $(0.5 \%)$ in the REG-EX group developed significant clinical evidence of ischemia prior to the administration of REG with two of the three patients receiving REG. One patient was diagnosed with an acute coronary syndrome and one with acute myocardial infarction $(0.3 \%)$. None of the patients randomized to REG-R developed a serious adverse cardiac event.

Based on the results of this trial, it would appear that performing REG imaging in the recovery phase of a submaximal ETT vs REG at rest is feasible with no significant reduction in ischemia detection, no major adverse effects on hemodynamics, a limited reduction in overall side effects, and an improvement in target-to- background ratio (but which apparently did not translate into improved image quality). REG-EX appears to be safe with a very low incidence of serious adverse cardiac events $(0.3 \%)$. Of note both patients with a cardiac event in the exercise group had evidence of ischemia prior to the administration of REG. The moral to this story is there is no value and potential additional harm to administering REG to a patient who has already demonstrated clinical evidence of ischemia. It is important to note that approximately $5-10 \%$ of patients will only develop electrocardiographic ischemia in the recovery phase of an exercise treadmill test and this finding is associated with ischemia by SPECT (indicative of significant coronary artery disease $)^{4}$ and an adverse outcome comparable to that of patients who have ischemia with exercise. ${ }^{5}$ In this regard, the 3 minute window from termination of exercise to administration of REG proposed in this study seems prudent and would allow one to identify such patients in whom REG should not be given.

\section{THE EXERRT TRIAL: ADDITIONAL OBSERVATIONS}

\section{Study Population and Influence on Results}

The baseline characteristics of the study population were similar between the two randomized groups with a mean age of 62 years and approximately $42 \%$ women. A similar and large percentage of patients had significant risk factors for CAD and prior myocardial infarction $(23 \%)$, coronary artery disease $(56 \%)$, and coronary revascularization $(45 \%)$. The primary endpoint of this study was to determine non-inferiority of serial REGEX/REG-R studies versus serial REG-R studies for detecting ischemia. This endpoint is interesting since there is no a priori reason why exercise should reduce detection of ischemia with REG. Exercise stress testing primarily induces endothelium-dependent changes in hyperemic flow, whereas vasodilator stress generally provokes endothelium-independent hyperemia. ${ }^{6,7}$ In this regard, exercise combined with REG might increase the disparity in myocardial blood flow in normal vs abnormally perfused vascular beds resulting in greater-not less-detection of perfusion abnormalities and ischemia. However, increasing hyperemic flows in myocardium supplied by normal coronary arteries might also lead to a paradoxical decrease in SPECT perfusion defects if the radiotracer being used cannot accurately track these changes. This is not a major issue with positron emission tomography tracers that have linear (O-15 water) or near-linear (N-13 Ammonia) extraction across all myocardial blood flows but could become an issue with agents that have relatively low extraction fractions at 
high flows such as technetium-99m-based SPECT tracers. In this scenario, the relatively low extraction fraction of technetium- $99 \mathrm{~m}$ at high myocardial flows (i.e., "rollover") could potentially reduce the relative difference in tracer counts between normal and hypoperfused myocardium leading to a reduction in perfusion defect size or even a normal study result. In the quantitative analysis performed from the ADVANCE trial by our group, REG induced a significant but relatively small increase in SPECT total perfusion defect size $(0.38 \% \mathrm{LV})$ as compared to adenosine even though adenosine induces hyperemic flows to considerably higher levels than REG (i.e., 3-3.5- vs 2-2.5-fold increase). ${ }^{8-11}$ This finding could have been secondary to the technetium "rollover" phenomenon and/or an increase in rate-pressure product produced by the higher heart rate achieved with REG vs adenosine. The data from EXERRT confirm a minimal effect of exercise with REG administration on initial SPECT results with $27 \%$ and $24 \%$ of patients having an abnormal REG-EX vs REG-R study and $7.2 \%$ and $5 \%$ of patients having ischemia, respectively.
Another interesting finding is the low incidence of both an abnormal study and ischemia on the baseline REG-EX and rest REG studies despite enrolling a large percentage of patients with known CAD so as to enrich detection of scintigraphic ischemia. Rozanski et al recently published a marked decline in the incidence of an abnormal stress MPI (40.9\% to $8.7 \%)$ and ischemia ( $29.6 \%$ to $5.0 \%$ ) over an 18-year period from 1991 to 2009 in 39,515 patients. ${ }^{12}$ The reason for this decline is multifactorial, but in patients with known CAD this could be modulated through coronary revascularization and the expanded use of anti-ischemic and statin medications. ${ }^{13,14}$ The presence of a normal study (74\%) or no/ minimal ischemia (94\%) in the current study was significantly higher than observed in the original ADVANCE studies where only $36 \%$ of patients had a normal initial study and $50 \%$ had ischemia. ${ }^{2}$ Although the current study mimics clinical practice, the large percentage of normal SPECT studies enhanced the ability to show non-inferiority between the randomized groups since a normal SPECT MPI can be achieved without the need of exercise and/or pharmacologic stressor agents.

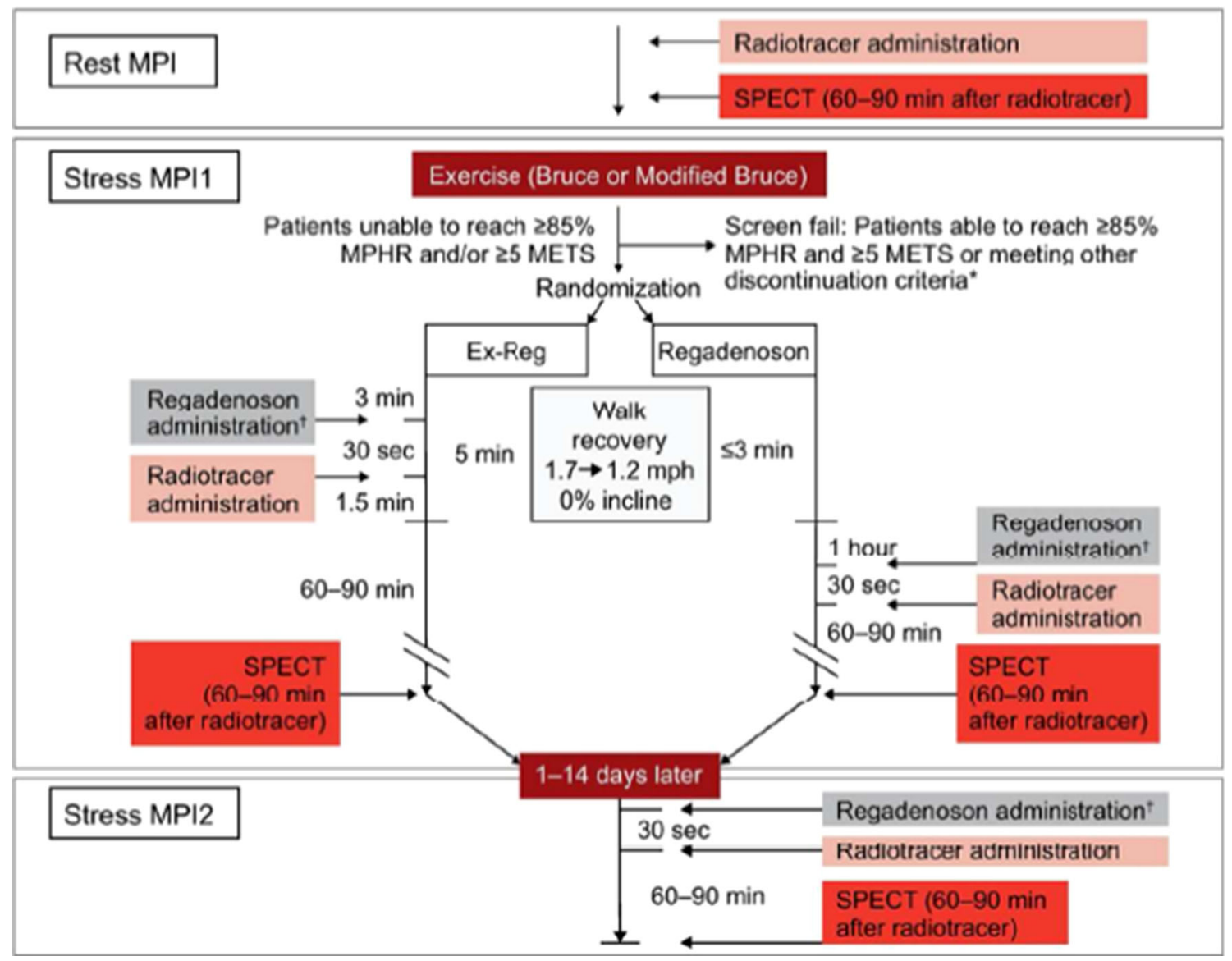

Fig. 1. EXERRT trial study design. 


\section{Differences in Test Results: MPI vs MPI 2}

Another intriguing finding was the greater detection of an abnormal study (28.5\% vs $26 \%$ ) and one with ischemia (9\% vs 6\%) on the second resting REG SPECT vs the initial study. Most (96\%) patients underwent a rest-stress imaging protocol on the initial randomized study, whereas all patients underwent stress first on the second study 1-14 days later with the initial rest study used for comparison. The authors also comment on this finding and suggest that "shine through" of the rest activity into the initial stress study may have reduced the ability to detect perfusion defects and/or ischemia. The authors suggest that when performing same-day reststress studies, a 1:4 (rather than the traditional 1:3) dosing ratio of technetium from the rest to stress study may be required but this would lead to yet a further increase in radiation exposure. An alternative option would be to perform a stress-rest imaging protocol with the advantage of performing stress-only imaging if the initial stress study is normal. ${ }^{15-18}$ This would reduce (not increase) patient and laboratory staff radiation exposure, improve laboratory throughput, reduce cost, conserve technetium, and add to patient satisfactionparticularly in view of the large percentage of patients as shown in this study who are likely to have a normal test result. ${ }^{15-18}$

\section{PREVIOUS STUDIES}

There have been 5 previous studies addressing the feasibility and safety of administering REG at peak exercise, ${ }^{19-21}$ at peak or low-level exercise, ${ }^{22}$ or at lowlevel exercise following peak $^{23}$ in a total of 1216 patients who could not attain $>85 \%$ of their predicted target heart rate. Two additional studies administered REG 1.5 minutes into low-level exercise $(1.7 \mathrm{mph}, 0 \%$ incline) for a total of 4 minutes. ${ }^{24,25}$ These studies generally enrolled patients with either a prior history of $\mathrm{CAD}$ and/or frequent comorbidities for CAD such as diabetes, hypertension, and hyperlipidemia (Table 1). Mean patient age was approximately 60 years and $1 / 3$ rd were women. The studies were either retrospective analyses or prospective non-randomized consecutive single-center patient series. Only one small $(n=75)$ single-center study randomized patients to either REG at peak exercise or at rest. ${ }^{21}$ These authors concluded that REG at peak stress was well tolerated and yielded similar scintigraphic results and image quality. The study by Thompson et al showed no advantage to administering REG at peak vs low-level exercise in the recovery phase (as performed in the current study) and there were less dramatic swings in individual systolic BP readings with low-level exercise. ${ }^{22}$ Other studies,

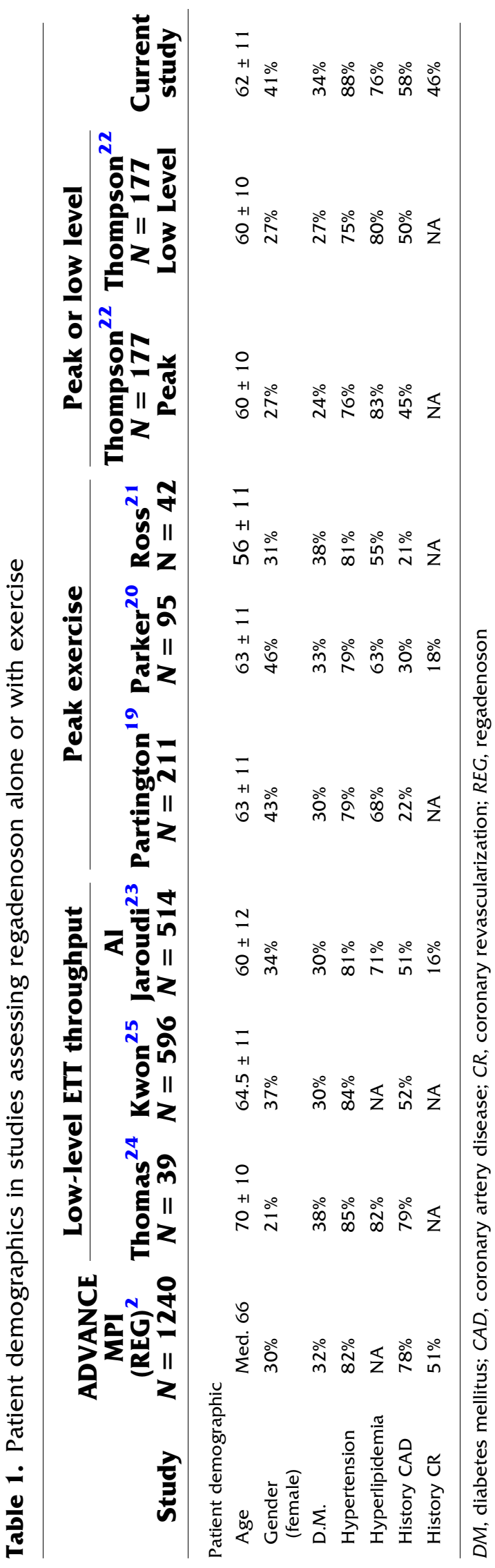


Table 2. Side effects observed in studies assessing regadenoson alone or with exercise

\begin{tabular}{|c|c|c|c|c|c|c|c|c|c|c|c|}
\hline \multirow[b]{2}{*}{ Side Effects } & \multirow[b]{2}{*}{$\begin{array}{c}\text { ADVANCE MPI } \\
(\text { REG) })^{2} \\
\mathrm{~N}=517\end{array}$} & \multicolumn{3}{|c|}{ Low Level ETT Throughout } & \multicolumn{3}{|c|}{ Peak Exercise } & \multicolumn{2}{|c|}{ Peak or Low Level } & \multirow[b]{2}{*}{\begin{tabular}{|c|} 
Current Study \\
Thomas \\
$\mathrm{N}=1142$ \\
\end{tabular}} & \multirow[b]{2}{*}{ Total } \\
\hline & & $\begin{array}{l}\text { Thomas }^{24} \\
\mathrm{~N}=39\end{array}$ & $\begin{array}{l}\text { Kwon }^{25} \\
N=596\end{array}$ & $\begin{array}{c}\text { Al Jaroudi } \\
N=514\end{array}$ & $\begin{array}{l}\text { Partington }^{19} \\
\mathrm{~N}=211\end{array}$ & $\begin{array}{c}\text { Parker }^{20} \\
\mathrm{~N}=95\end{array}$ & $\begin{array}{l}\text { Ross }^{21} \\
\mathrm{~N}=42\end{array}$ & $\begin{array}{c}\text { Thompson }^{22} \\
\mathrm{~N}=177 \\
\text { Peak }\end{array}$ & $\begin{array}{c}\text { Thompson } 22 \\
\mathrm{~N}=177 \\
\text { Low Level }\end{array}$ & & \\
\hline SOB & $25 \%$ & $54 \%$ & $23 \%$ & $12 \%$ & \multirow{5}{*}{$\begin{array}{r}24 \% \\
10.4 \%\end{array}$} & $83 \%$ & NA & $60 \%$ & $87 \%$ & $25 \%$ & $25 \%$ \\
\hline CP & $27 \%$ & $10 \%$ & $33 \%$ & $13 \%$ & & $39 \%$ & NA & $15 \%$ & $22 \%$ & $6 \%$ & $14 \%$ \\
\hline Headache & $29 \%$ & $23 \%$ & $0.6 \%$ & $0.4 \%$ & & $22 \%$ & NA & NA & NA & $15 \%$ & $9.8 \%$ \\
\hline Flushing & $17 \%$ & $13 \%$ & NA & NA & & $26 \%$ & NA & $3 \%$ & $9 \%$ & $8 \%$ & $6 \%$ \\
\hline Dizziness & $7 \%$ & $13 \%$ & $12 \%$ & $7 \%$ & & $39 \%$ & NA & $15 \%$ & $11 \%$ & $19 \%$ & $11 \%$ \\
\hline GI Symptoms & $12 \%$ & $13 \%$ & $1.2 \%$ & $1.9 \%$ & NA & $37 \%$ & NA & $4.5 \%$ & $12 \%$ & $13 \%$ & $6 \%$ \\
\hline High degree AV Block & $0 \%$ & $0 \%$ & $0.3 \%$ & $0 \%$ & $0 \%$ & NA & $0 \%$ & $0 \%$ & $0 \%$ & $0 \%$ & $0.07 \%$ \\
\hline Aminophylline Use & NA & NA & $0.7 \%$ & $0.8 \%$ & $0.5 \%$ & NA & NA & NA & NA & NA & $0.7 \%$ \\
\hline MI/NCS & $0 \%$ & $0 \%$ & $0 \%$ & $0 \%$ & $0 \%$ & $0 \%$ & $0 \%$ & $0 \%$ & $0 \%$ & $0.4 \%$ & $0.07 \%$ \\
\hline Cardiac Arrest & $0 \%$ & $0 \%$ & $0 \%$ & $0 \%$ & $0 \%$ & $0 \%$ & $0 \%$ & $0 \%$ & $0 \%$ & $0 \%$ & \\
\hline Hypotension & & SBP $<1003 \%$ & $>30 \mathrm{~mm} \downarrow 12 \%$ & $>30 \mathrm{~mm} \downarrow 12 \%$ & SBP $<1001.4 \%$ & NA & $>20 \mathrm{~mm} \downarrow 0 \%$ & $>20 \mathrm{~mm} \downarrow 16 \%$ & $7 \%$ & $>35 \mathrm{~mm} \downarrow 29 \%$ & \\
\hline Hypertension & & NA & NA & NA & $>10 \mathrm{~mm} \uparrow \mathrm{SBP} 12 \%$ & NA $>$ & $>20 \mathrm{~mm} \uparrow 0 \%$ & $>20 \mathrm{~mm} \uparrow 22 \%$ & $10 \%$ & $>50 \mathrm{~mm} \uparrow 1.9 \%$ & \\
\hline
\end{tabular}

$S O B$, shortness of breath; $C P$, chest pain; $G I$, gastrointestinal; $A V$, atrioventricular; $M I$, myocardial infarction; $A C S$, acute coronary syndrome; $R E G$, regadenoson

including the current trial, have demonstrated small mean changes in systolic BP following REG administration with a minority of patients developing significant hypotension $\left(\mathrm{SBP}<100 \mathrm{mmHg}\right.$ in $1.8 \%^{19,23}$ ) or hypertension $\left(\mathrm{SBP}>10 \mathrm{~mm}\right.$ rise in $12 \%{ }^{19}$ or $>20 \mathrm{~mm} \mathrm{Hg}$ rise in $9 \%){ }^{22,23}$ In the current study, only $1.9 \%$ of patients had a drop in SBP to $<90 \mathrm{mmHg}$ and this was less than that observed with rest REG administration (3.3\%). Regarding side effects, a REG-EX protocol did not significantly decrease the incidence of chest pain or shortness of breath in prior studies ${ }^{19,22}$ or the current one, but there were significant decreases in those side effects most typically associated with REG including headache, dizziness, flushing, and gastrointestinal symptoms (Table 2). ${ }^{19,22}$ In the study by Partington et al, the latter symptoms occurred in $49 \%$ of patients receiving REG-R vs $6 \%$ of those during REG-EX. ${ }^{19}$ In the current study, $10 \%$ fewer patients developed these side effects with REG-EX versus REG-R. Finally, as in the current study, target-(heart)-to-background ratios improved when REG was combined with exercise but with no clear improvement in study quality or interpretability. This has likewise been shown in other studies to varying degrees.

\section{DOES EXERRT MOVE THE NEEDLE?}

As noted above, there have been many smaller primarily non-randomized studies which have addressed the issue of administering REG while a patient is recovering from low-level exercise, and the sum of data indicates that this is a safe practice. The option of administering REG in the recovery phase of an exercise test allows patients the opportunity to attempt a maximal exercise test among those in whom their physical capabilities are unknown. EXERRT shows no difference in SPECT MPI results when REG is administered with exercise, no untoward hemodynamic effects that would warrant concern, and a reduction in common REGinduced side effects. Despite these potential advantages, the authors appropriately recommend not administering REG to patients who have already declared themselves ischemic based on the exercise test alone. Previous studies had excluded patients with exercise-induced ischemia and with no cardiac events reported. From a clinical perspective, this would seem a wise decision. EXERRT is a well-designed, rigorous trial showing the feasibility and safety of administering REG following submaximal exercise testing and should prove to be the final word regarding this issue.

\section{Disclosure}

Author is a Consultant as well as on the Speaker Bureau for Astellas, Inc.

\section{References}

1. Prenner BM, Bukofzer S, Behm S, Feaheny K, McNutt BE. A randomized, double-blind, placebo-controlled study assessing the safety and tolerability of regadenoson in subjects with asthma or chronic obstructive pulmonary disease. J Nucl Cardiol 2012;19:681-92.

2. Cerqueira MD, Nguyen $P$, Staehr P, Underwood SR, Iskandrian AE. ADVANCE-MPI Trial Investigators. Effects of age, gender, obesity, and diabetes on the efficacy and safety of the selective A2A agonist regadenoson versus adenosine in myocardial perfusion imaging integrated ADVANCE-MPI trial results. JACC Cardiovasc Imaging 2008; 1:307-16.

3. Iskandrian AE, Bateman TM, Belardinelli L, Blackburn B, Cerqueira MD, Hendel RC, Lieu H, Mahmarian JJ, Olmsted A, Underwood SR, Vitola J, Wang W, ADVANCE MPI Investigators. Adenosine versus regadenoson comparative evaluation in myocardial perfusion imaging: results of the ADVANCE phase 3 multicenter international trial. J Nucl Cardiol 2007;14:645-58.

4. Soto JR, Watson DD, Beller GA. Incidence and significance of ischemic ST-segment depression occurring solely during recovery after exercise testing. Am J Cardiol 2001;88:670-2. 
5. Rywik TM, Zink RC, Gittings NS, Khan AA, Wright JG, O'Connor FC, et al. Independent prognostic significance of ischemic ST-segment response limited to recovery from treadmill exercise in asymptomatic subjects. Circulation 1998;97:2117-22.

6. Buus NH, Bottcher M, Hermansen F, Sander M, Nielsen TT, Mulvany MJ. Influence of nitric oxide synthase and adrenergic inhibition on adenosine-induced myocardial hyperemia. Circulation 2001;104:2305-10.

7. Duncker DJ, Bache RJ. Regulation of coronary blood flow during exercise. Physiol Rev 2008;88:1009-86.

8. Mahmarian JJ, Peterson LE, Xu J, Cerqueira MD, Iskandrian AE, Bateman TM, et al. Regadenoson provides perfusion results comparable to adenosine in heterogeneous patient populations: a quantitative analysis from the ADVANCE MPI trials. J Nucl Cardiol 2015;22:248-61.

9. Wilson RF, Wyche K, Christensen BV, Zimmer S, Laxson DD. Effects of adenosine on human coronary arterial circulation. Circulation 1990;82:1595-606.

10. Belardinelli L, Shryock JC, Snowdy S, Zhang Y, Monopoli A, Lozza $\mathrm{G}$, et al. The A2A adenosine receptor mediates coronary vasodilation. J Pharmacol Exp Ther 1998;284:1066-73.

11. Lieu HD, Shyock JC, von Mering GO, Gordi T, Blackburn B, Olmsted AW, et al. Regadenoson, a selective A2A adenosine receptor agonist, causes dose-dependent increases in coronary blood flow velocity in humans. J Nucl Cardiol 2007;14:51420.

12. Rozanski A, Gransar H, Hayes SW, Min J, Friedman JD, et al. Temporal trends in the frequency of inducible myocardial ischemia during cardiac stress testing: 1991-2009. J Am Coll Cardiol 2013;61:1054-65.

13. Mahmarian JJ, Dakik HA, Filipchuk NG, Shaw LJ, lskander SS, Ruddy TD, Keng F, Henzlova MJ, Allam A, Moye LA, Pratt CM, for the ADENOSINE SESTAMIBI SPECT POST-INFARCTION EVALUATION (INSPIRE) Investigators. An initial strategy of intensive medical therapy is comparable to that of coronary revascularization for suppression of scintigraphic ischemia in high risk but stable survivors of acute myocardial infarction. J Am Coll Cardiol 2006;48:2458-67.

14. Mahmarian JJ. Monitoring medical therapy: The role of noninvasive imaging. In: Dilsizian V, Narula J, Braunwald E, editors. Atlas of Nuclear Cardiology. 2nd ed. Philadelphia: Current Medicine; 2006. p. 191-210.

15. Chang SM, Nabi F, Xu J, Raza U, Mahmarian JJ. Normal stressonly versus standard stress/rest myocardial perfusion imaging: similar patient mortality with reduced radiation exposure. J Am Coll Cardiol 2010;55:221-30.
16. Mahmarian JJ. Stress only myocardial perfusion imaging: Is it time for a change? [editorial]. J Nucl Cardiol 2010;17:529-35.

17. Nabi F, Kassi M, Muhyieddeen K, Chang SM, Xu J, Peterson LE, Wray NP, Shirkey BA, Ashton CM, Mahmarian JJ. Optimizing evaluation of patients with low-to-intermediate-risk acute chest pain: a randomized study comparing stress myocardial perfusion tomography incorporating stress-only imaging versus cardiac CT. J Nucl Med 2016;57:378-84

18. Duvall WL, Guma KA, Kamen J, Croft LB, Parides M, George T, Henzlova MJ. Reduction in occupational and patient radiation exposure from myocardial perfusion imaging: impact of stressonly imaging and high-efficiency SPECT camera technology. J Nucl Med 2013;54:1251-7.

19. Partington SL, Lanka V, Hainer J, Blankstein R, Skali H, Forman $\mathrm{DE}$, et al. Safety and feasibility of regadenoson use for suboptimal heart rate response during symptom-limited standard Bruce exercise stress test. J Nucl Cardiol 2012;19:970-8.

20. Parker MW, Morales DC, Slim HB, Ahlberg AW, Katten DM, Cyr $\mathrm{G}$, et al. A strategy of symptom-limited exercise with regadenoson-as-needed for stress myocardial perfusion imaging: a randomized controlled trial. J Nucl Cardiol 2013;20:185-96.

21. Ross MI, Wu E, Wilkins JT, Gupta D, Shen S, Aulwes D, et al. Safety and feasibility of adjunctive regadenoson injection at peak exercise during exercise myocardial perfusion imaging: the both exercise and regadenoson stress test (BERST) trial. J Nucl Cardiol 2013;20:197-204

22. Thompson RC, Patil H, Thompson EC, Thomas GS, Al-Amoodi M, Kennedy KF, et al. Regadenoson pharmacologic stress for myocardial perfusion imaging: a three-way comparison between regadenoson administered at peak exercise, during walk recovery, or no-exercise. J Nucl Cardiol 2013;20:214-21.

23. Al Jaroudi WA, Alraies MC, Cerquiera MD, Jaber WA. Safety and tolerability of regadenoson in 514 SPECT MPI patients with and without coronary artery disease and submaximal exercise heart rate response. Eur J Nucl Med Mol Imaging 2013;40:341-8.

24. Thomas GS, Thompson RC, Miyamoto MI, Ip TK, Rice DL, Milikien D, et al. The RegEx trial: a randomized, double-blind, placebo- and active-controlled pilot study combining regadenoson, a selective A (2A) adenosine agonist, with low-level exercise, in patients undergoing myocardial perfusion imaging. J Nucl Cardiol 2009; 16:63-72.

25. Kwon DH, Cerqueira MD, Young R, Houghtaling P, Lieber E, Menon V, et al. Lessons from regadenoson and low-level treadmill/regadenoson myocardial perfusion imaging: initial clinical experience in 1263 patients. J Nucl Cardiol 2010;17:853-7. 